\title{
Diagnosis and analysis of a sebaceous gland tumour of the external acoustic meatus in a Cocker Spaniel dog
}

\author{
Jiashi Dong, Chunling Fan, Dingxin Liu, Peng Li \\ Heilongjiang Bayi Agricultural University, College of Animal Science and Technology, Heilongjiang Daqing, China
}

Received May 6, 2020

Accepted February 24, 2021

\begin{abstract}
Sebaceous gland tumour (SGT) is a rare tumour with a low incidence rate, which mainly occurs in the eyelid and periocular sebaceous gland. In 2019, our laboratory received a case involving a sebaceous gland tumour of the external auditory meatus for pathological diagnosis. It is a rare malignant tumour in the whole body. Data regarding a sebaceous gland tumour in the dog's external auditory meatus are few. This case is instructive for veterinary diagnosis and treatment. The dog recovered well after surgery at the outpatient department, and no recurrence was observed in the follow-up visit one year later. This study will help the identification of SGT in Spaniel dogs.
\end{abstract}

Canine, ear, surgery

Sebaceous gland tumour (SGT) is a rare, aggressive, and highly malignant cutaneous tumour accounting for less than $1 \%$ of all cutaneous malignant tumours. It is characterized by a low incidence rate and high mortality. Sebaceous skin tumours are classified into sebaceous adenoma, sebaceoma, and sebaceous carcinoma (Flux 2017). In human medicine, SGT dominantly affects the periocular sebaceous glands (Manteghi et al. 2014).

This malignancy can arise from the sebaceous units in the tarsus (meibomian glands), in association with the cilia (Zeis glands), brow, and caruncle (Shields et al. 2015). In human medicine, both architectural and cytologic characteristics are used to distinguish benign sebaceous neoplasms from malignant ones (Tirado et al. 2019). Histological and immunohistochemical characteristics are often challenging to diagnose (Schmitz et al. 2017). Sebaceous carcinoma may masquerade for years as an inflammatory condition (Gonzalez-Fernandez et al. 1998), its clinical presentation may be non-specific, and a biopsy is important to establish a diagnosis and to differentiate from mimickers including benign sebaceous neoplasms, other adnexal tumours, and basal cell carcinoma (Knackstedt and Samie 2017). Early identification and appropriate treatment may help improve the prognosis (Cicinelli and Kaliki 2019) but there is still a certain recurrence rate. Some veterinarians report that cryotherapy can be used to treat SGT. However, surgical removal and histopathological examination is be necessary in the treatment of SGT (Angileri et al. 2020).

Common tumours of the external acoustic meatus in dogs include cerumen adenoadenoma, inflammatory polyp, papilloma, basal cell tumour, cerumen adenocarcinoma, squamous cell carcinoma and tumour of unknown origin (Withrow and Vail 2007). This study reports a case of a SGT of the dog's external acoustic meatus.

\section{Case description}

In 2019, a case of SGT of the external acoustic meatus was presented and pathological diagnosis was performed. This case was a 12-year-old male Cocker Spaniel with the body

Address for correspondence:

Peng $\mathrm{Li}$

College of Animal Science and Technology

Heilongjiang Bayi Agricultural University

Heilongjiang Daqing, China 163319

Phone: +8613836962800

E-mail: lipeng98@163.com

http://actavet.vfu.cz/ 
temperature of $37.7^{\circ} \mathrm{C}$ and the body weight of $12 \mathrm{~kg}$, which was immunized. We found bilateral ear meatus hyperplasia of the size of a table-tennis ball and irregular margin, with a strong odour and liquefaction in the middle of the tumour (Plate VII, Fig. 1A,B). The mental state, appetite, and desire to drink were normal.

Upon initial diagnosis, the tumour of the external acoustic meatus was treated by total resection of the external acoustic meatus. Upon resection, the tumour was cryopreserved at $-80{ }^{\circ} \mathrm{C}$. Samples of $2 \mathrm{~mm}$ thickness were taken from the excised tissues and made into pathological sections by alcohol dehydration, xylene transparency, and other operations.

Pathological examination showed that subcutaneous sebaceous gland cells proliferated, and the boundary between tumour cells and normal cells was unclear. There were obvious carcinomatous nests and carcinomatous beads with a slight left shift of the nucleus as shown in Fig. 2 (Plate VII). The dog recovered well after surgery at the outpatient department (Plate VIII, Fig. 3A,B), and no recurrence was found at the follow-up visit one year later.

\section{Discussion}

Under long-term stimulation, inflammation and mites in the dog's external acoustic meatus will generally cause hyperplasia of the meatus. Severe hyperplasia can block the external acoustic meatus and affect the dog's hearing. The formation of anoxic environment in the occluded meatus will further aggravate the occurrence of otitis externa, especially the infection of anaerobic bacteria, increasing the incidence of tumours in the external acoustic meatus. Therefore, treatment should be conducted in time after the occurrence of otitis externa or occlusion of the external acoustic meatus. The occurrence of tumours in the external acoustic meatus is related to the family history of tumours (Ma et al. 2019).

At present, the best treatment for dog's external acoustic meatus hyperplasia and tumour is surgical treatment, which is also used for serious mites, although there are a few cases of external ear benign tumour recurring after the operation. The common tumours in the external acoustic meatus of dogs include adenoma of the cerumen gland, inflammatory polyp, papilloma, basal cell tumour, adenocarcinoma of the cerumen gland and squamous cell carcinoma. Sebaceous carcinoma is a relatively uncommon malignant epithelial neoplasm with a predilection for the periocular region (Plaza et al. 2015).

Although the pathogenesis of SGT is unknown, its histogenesis may be related to the stimulation of long-term purulent secretion, viral infection, radiation effects (Misago and Toda 2016), and other secondary factors such as myeloid sarcoma invasion (Jo et al. 2019). The dog's ear meatus tumour may be related to ear mites; the patient's external acoustic meatus with chronic otitis had not been cleaned for many years. Because no other tumours occurred in the patient, this tumour case may have been caused by the above two points. Tumours of the external acoustic meatus are usually treated by resection of the external acoustic meatus. Although this case showed a slight malignant change in the tissue section, recurrence did not occur within one year after the operation, and the effect of the treatment was obvious.

This case illustrated that a low-grade malignancy tumour in dogs can be treated by surgery with no recurrence in a short period of time without radiotherapy. Since the followup visit of this case was interrupted, it is unknown whether the disease will recur in the long term. Because the life span of dogs is much shorter than that of humans, it is unclear how long senior dogs can survive with malignant tumours.

The pathological section of this case showed multiple areas of hyperplastic sebaceous glands, and the boundary between tumour tissue and normal tissue was blurred. The tumour was considered to be mildly malignant and this picture can be used as a guide for the study of canine SGT for the external acoustic meatus. This case provided rich data of sebaceous adenocarcinoma of the external acoustic meatus. 


\section{References}

Angileri M, Furlanello T, De Lucia M 2020: Cryotherapy to treat benign skin tumours in conscious dogs. Vet Dermatol 31: 163-166

Cicinelli MV, Kaliki S 2019: Ocular sebaceous gland carcinoma: an update of the literature. Int Ophthalmol 39: 1187-1197

Flux K 2017: Sebaceous neoplasms. Surg Pathol Clin 10: 367-382

Gonzalez-Fernandez F, Kaltreider SA, Patnaik BD, Retief, Y Bao, S Newman, M H Stoler, P A Levine 1998: Sebaceous carcinoma. Tumor progression through mutational inactivation of p53. Ophthalmology 105: 497-506

Jo S, Shim HK, Kim JY, Bae SK, Kim MR 2019: Myeloid sarcoma that infiltrated a preexisting sebaceous lymphadenoma in the parotid gland: diagnostic challenges and literature review. Biomed Res Int 9869406

Knackstedt T, Samie FH 2017: Sebaceous carcinoma: a review of the scientific literature. Curr Treat Options Oncol 18: 47

Manteghi A, Zwillenberg S, Arguello-Guerra V 2014: Sebaceous carcinoma of the parotid gland: a case report and review of the literature. Ear Nose Throat J 93: E29-E32

Ma S, Li LT, Ma YJ, Shi JT, Zhou CF, Liu YM, Chen J 2019: Preliminary analysis of the influencing factors of external auditory canal cancer secondary to radiotherapy for nasopharyngeal carcinoma. Chinese Journal of Radiation Oncology 09: 657-659

Misago N, Toda S 2016: Sebaceous carcinoma within rippled/carcinoid pattern sebaceoma. J Cutan Pathol 43: 64-70

Plaza JA, Mackinnon A, Carrillo L, Prieto VG, Sangueza M, Suster S 2015: Role of immunohistochemistry in the diagnosis of sebaceous carcinoma: a clinicopathologic and immunohistochemical study. Am J Dermatopathol 37: 809-821

Schmitz EJ, Herwig-Carl MC, Holz FG, Loeffler KU 2017: Sebaceous gland carcinoma of the ocular adnexa - variability in clinical and histological appearance with analysis of immunohistochemical staining patterns Graefes Arch Clin Exp Ophthalmol 255: 2277-2285

Shields JA, Saktanasate J, Lally SE, Carrasco JR, Shields CL 2015: Sebaceous carcinoma of the ocular region: The 2014 Professor Winifred Mao lecture. Asia Pac J Ophthalmol (Phila) 4: 221-227

Tirado M, Metze D, Sahlmann J, Böer-Auer A 2019: Cytologic grading of cutaneous sebaceous neoplasms: does it help to differentiate benign from malignant? Am J Dermatopathol 41: 722-732

Withrow SJ, Vail DM 2007: Withrow \& MacEwen's Small Animal Clinical Oncology. Fourth edn. Elsevier, St. Louis, Missouri, USA. 864 p. 
Plate VII

Jiashi D. et al.: Diagnosis ... pp. 87-89

A

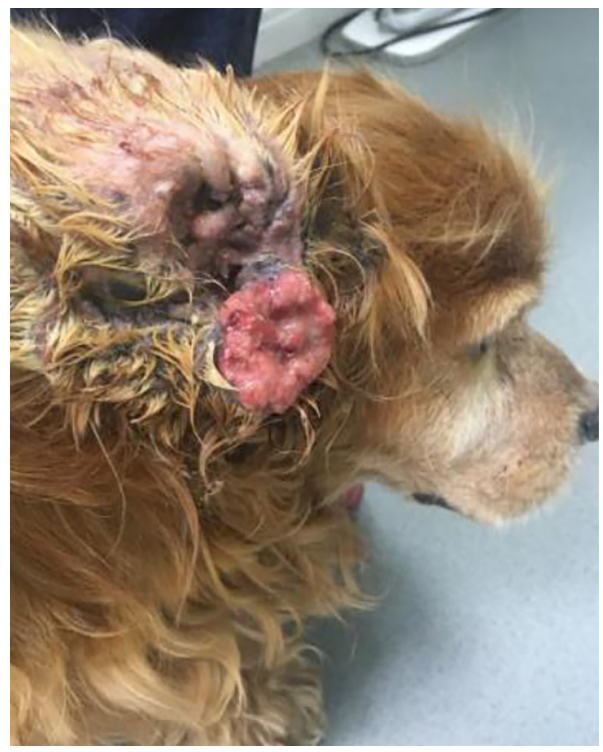

B

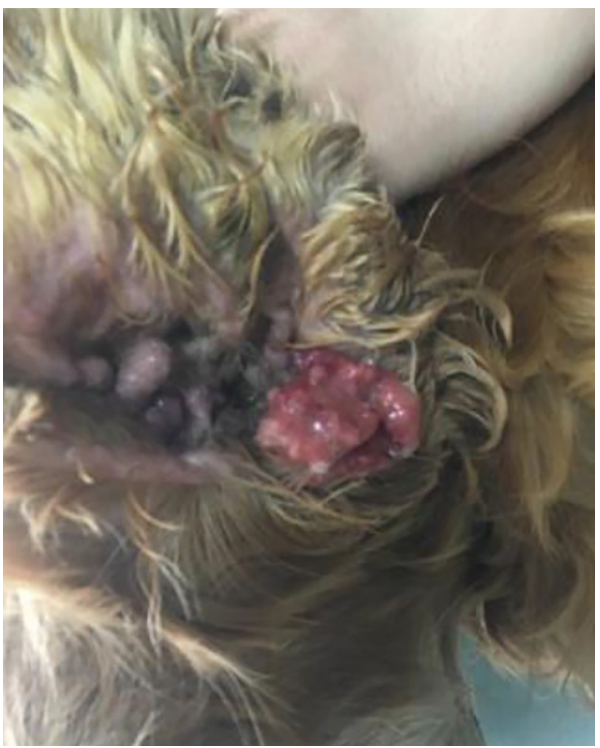

Fig.1. A: Right external acoustic meatus tumour. B: Left external acoustic meatus tumour.

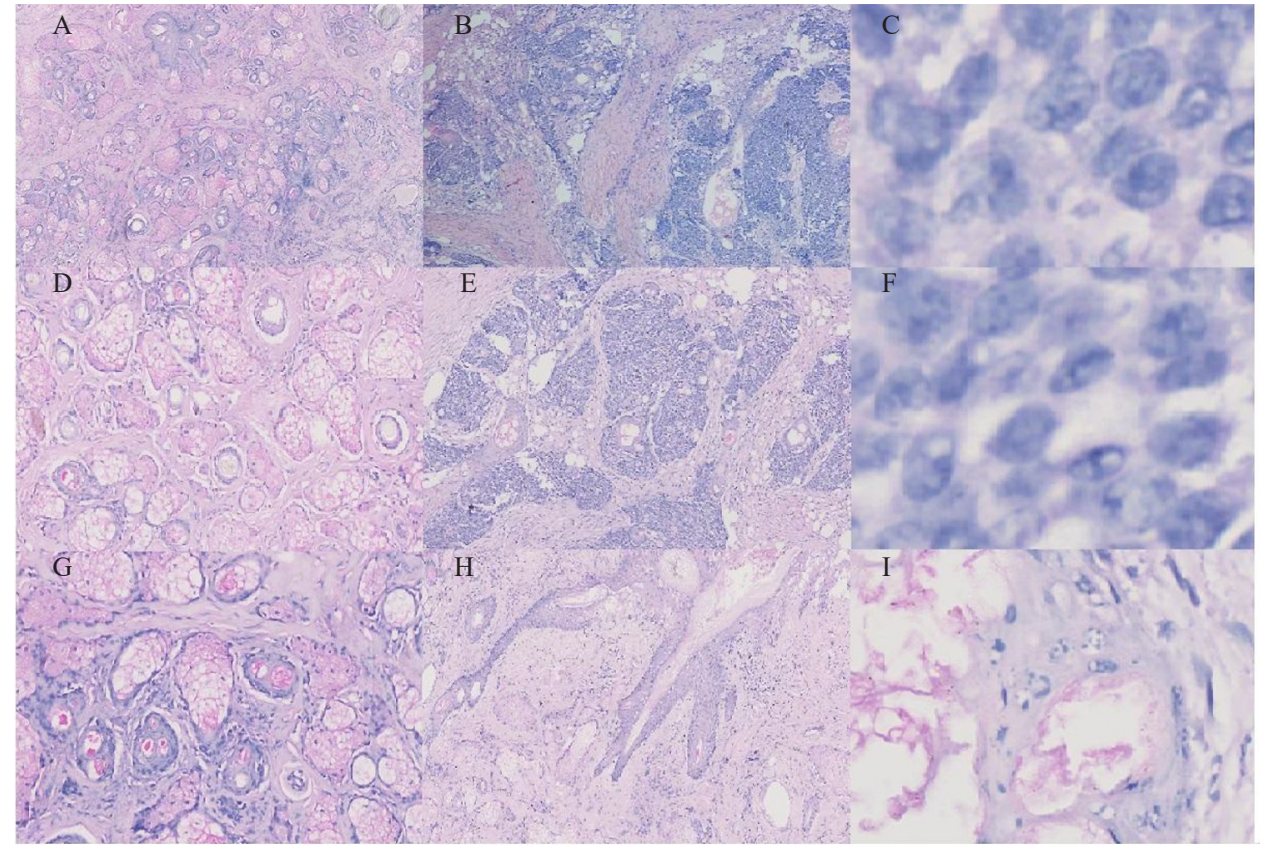

Fig. 2. Pathological section. A, B, and C: multiple areas of hyperplastic sebaceous glands, H\&E, $\times 200$. D, E, and F: proliferated tumour nests and the unclear boundary of the tissue, H\&E, $\times 200$. G, H, and I: two-lobed nucleus, $\mathrm{H} \& \mathrm{E}, \times 400$. 
A

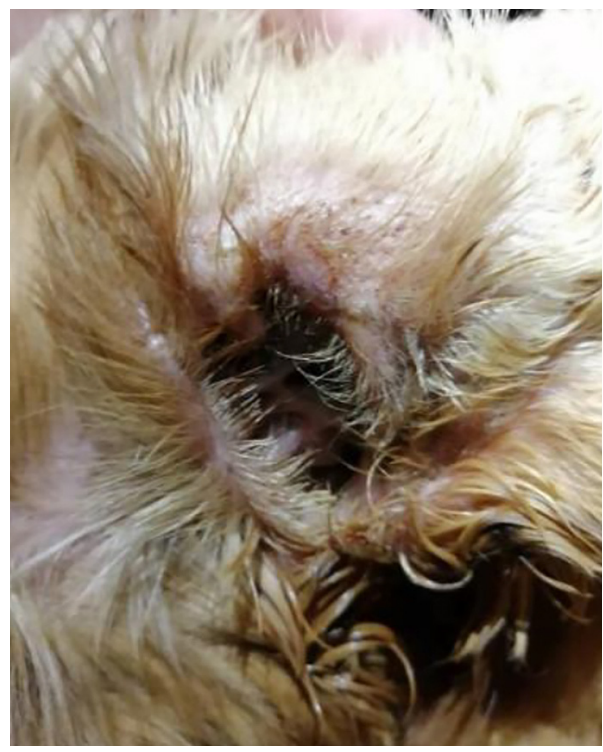

Fig. 3. Left (A) and right (B) ear after healing.
B

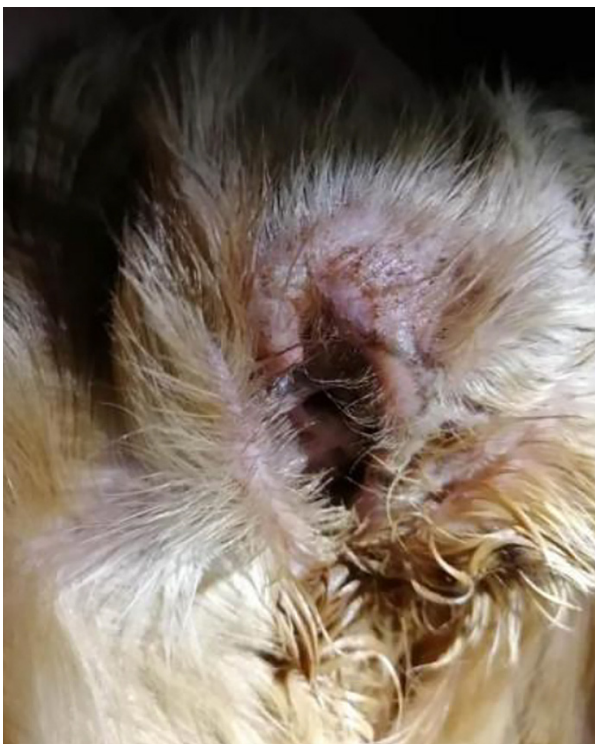

